\title{
Polycarbonate Polymer Surface Modification by Extreme Ultraviolet (EUV) Radiation
}

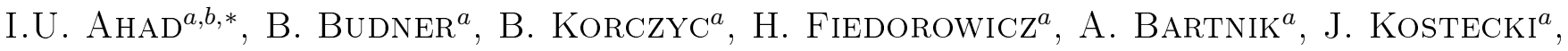 \\ S. BURDYŃSKA ${ }^{a}$ AND D. BRABAZON ${ }^{b}$
}

${ }^{a}$ Institute of Optoelectronics, Military University of Technology, S. Kaliskiego 2, 00-908 Warszawa, Poland ${ }^{b}$ Advanced Processing Technology Research Centre, School of Mechanical and Manufacturing Engineering Faculty of Engineering \& Computing, Dublin City University, Dublin 9, Ireland

\begin{abstract}
The degree of the biocompatibility of polycarbonate (PC) polymer used as biomaterial can be controlled by surface modification for various biomedical engineering applications. In the past, PC samples were treated by excimer laser for surface reorganization however associated process alteration of bulk properties is reported. Extreme ultraviolet radiation can be employed in order to avoid bulk material alteration due to its limited penetration. In this study, a $10 \mathrm{~Hz}$ laser-plasma EUV source based on a double-stream gas-puff target irradiated with a $3 \mathrm{~ns}$ and $0.8 \mathrm{~J}$ Nd:YAG laser pulse was used to irradiate PC samples. The PC samples were irradiated with different number of EUV shots. Pristine and EUV treated samples were investigated by scanning electron microscopy and atomic force microscopy for detailed morphological characterization of micropatterns introduced by the EUV irradiation. Associated chemical modifications were investigated by X-ray photoelectron spectroscopy. Pronounced wall-type micro- and nanostructures appeared on the EUV modified surface resulting in a change of surface roughness and wettability.
\end{abstract}

DOI: $10.12693 /$ APhysPolA.125.924

PACS: 81.05.Lg, 81.40.Wx, 81.16.Rf, 81.65.-b

\section{Introduction}

Organic polymers are considered as important materials in various biomedical applications ranging from conventional cell growth to construction of hybrid tissues and artificial organs. Synthetic and naturally occurring polymers are now becoming an important element in new strategies for producing engineered tissues. Several classes of polymers are now employed in biomedical applications, including situations in which the polymer remains in intimate contact with cells and tissues for prolonged periods [1-4]. Yet these polymers very often do not possess the surface properties needed for these applications, making it necessary to modify the polymer surface.

Among various techniques, excimer lasers have been used by various groups for surface modification of polymers [5-8]. However the degradation of bulk material is reported due to a higher penetration depth of excimer lasers in organic polymers. Laser ablation leans on the nature of the material and its ability to absorb energy. Therefore the wavelength of the ablation laser should be chosen to have a minimum absorption depth. Laser ablation rate largely depends upon laser wavelength and pulse length. Ablation rate also relies upon the amount of total energy delivered in one shot and optimal spectral distribution. Since EUV radiation has limited absorption depth, it can be successfully employed for surface modi-

*corresponding author; e-mail: iuahad@wat.edu.pl fication with optimal results [9]. In a previous study by our group, improved biocompatibility was demonstrated from EUV irradiated polyethylene terephthalate (PET) samples [10]. Moreover doping of nitrogen in biomaterials by EUV surface modification has also been demonstrated [11].

Polycarbonates (PC) have been used as biomaterials with applications ranging from renal dialysis to cardiac surgery products however biocompatibility problems are reported. The wettability of PC can be controlled by introducing micro or nanopatterning on their surface using ultraviolet (UV) radiation in order to control the degree of the biocompatibility [8]. However UV radiation may also influence the bulk properties of treated materials as discussed above. For biomaterials, important physical (mechanical and structural) properties should be retained during surface modification for the biocompatibility control. In such a way, the implant material is able to provide proper mechanical functions with the tailored biocompatible surface. In this study the PC samples irradiated by a $10 \mathrm{~Hz}$ laser-plasma EUV source were specially dedicated for polymer processing. The PC samples were treated with different number of EUV laser pulses. Surface morphological, topographical and chemical modifications in EUV treated samples were characterized by scanning electron microscopy (SEM), atomic force microscopy (AFM), and X-ray photoelectron spectroscopy (XPS).

\section{Materials and methods}

Polymer samples were irradiated by a $10 \mathrm{~Hz}$ laser plasma based EUV source. This source is specially dedicated to polymer processing. The source is based on a 
double-stream gas-puff target, irradiated with a $3 \mathrm{~ns}$ and $0.8 \mathrm{~J} \mathrm{Nd}$ :YAG laser pulse. The gas-puff target system allows for debris free operation as compared to those of solid target systems [12]. The interaction chamber of the source is separated from the gas target irradiation chamber in order to have a differential pumping setup. The pressure in the target chamber is maintained at the few millibars whereas in the EUV-sample interaction chamber, the pressure is kept around $10^{-2}$ millibar. The polymer sample was placed on a moveable $X Y Z$ translation stage controlled by an application software based control panel. This allows irradiation of a particular area of the sample as per requirements. An auxiliary gas-puff valve is mounted in the interaction chamber in order to introduce an additional gas (such as helium or nitrogen). Detailed experimental setup and source information can be found in previous publications by our group [13, 14]. The PC samples were supplied by Goodfellow Cambridge Ltd. England with $0.06 \mathrm{~mm}$ thickness and were irradiated with 100, 200, 300, and 600 EUV shots (as shown in Fig. 1). The diameter of the trace of the EUV shots was around $1.5 \mathrm{~mm}$.

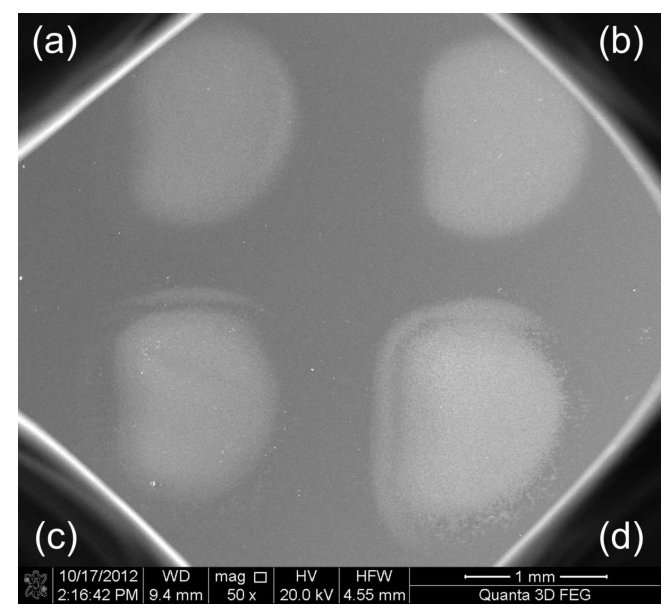

Fig. 1. SEM image of the PC sample irradiated with (a) 100, (b) 200, (c) 300, and (d) 600 EUV shots.

In a separate experimental setup, polycarbonate sample area of about $12 \mathrm{~mm} \times 10.5 \mathrm{~mm}$ was irradiated with EUV photons. This is achieved by the controlled translationary movements of the stage along the $X$ - and $Y$-planes so that $20 \mathrm{EUV}$ shots irradiated per $1.5 \mathrm{~mm}$ diameter of the polymer sample while the sample stage is moving. The stage is moved horizontally for $10 \mathrm{~mm}$ and then vertically for $1.5 \mathrm{~mm}$. This scheme is repeated 7 times. The resulting EUV modified polymer sample is shown in Fig. 2.

The helium gas is injected through the auxiliary valve during the EUV irradiation which helps to avoid optics degradation by the ablated material. EUV treated and pristine samples were characterized for surface modifications. For morphological studies, two SEM with different resolutions were used (VEGA II SBU by TESCAN Czech Republic and Quanta 3D FEG by FEI USA). Atomic

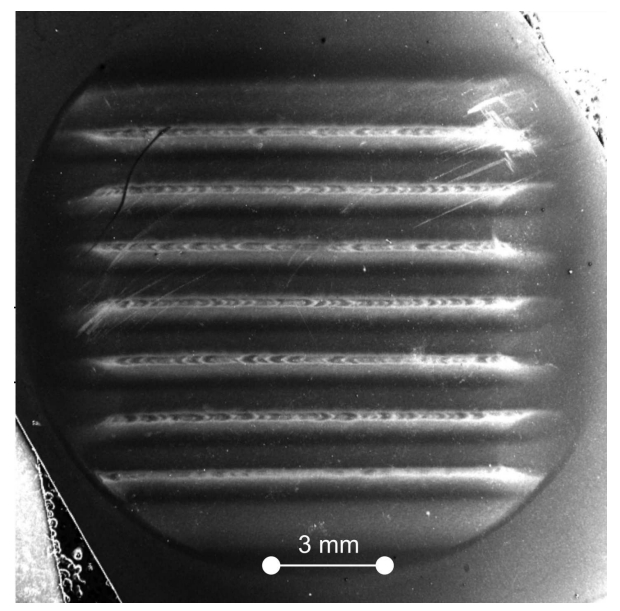

Fig. 2. SEM image of the PC sample irradiated with 20 EUV shots with sample holder moving at $0.5 \mathrm{~mm} / \mathrm{s}$.

force microscopes supplied by Veeco, USA and NT-MDT, Russia were used. Images were acquired at different resolution, magnification and scale as per requirement in order to investigate the micro- and nanostructures which appeared on the EUV treated surfaces. In order to investigate the height of these structures AFM was used in semi-contact mode and the acquired images were processed using an image analysis tool developed by NT-MDT. Low resolution X-ray photoelectron spectroscopy (XPS) scans were obtained to investigate the chemical modifications in the EUV treated samples. The $\mathrm{C} 1 \mathrm{~s}$ peak was calibrated by shifting to $285 \mathrm{eV}$ for measured samples in order to compensate the effects of sample charging and flood gun.

\section{Results and discussion}

A single polycarbonate sample was irradiated with 100 , 200, 300, and 600 EUV shots at different places to visualize the EUV trace difference as shown in Fig. 1. Figure 3a and $b$ shows 10,000 times magnified SEM images of PC sample irradiated with 200 and 600 shots, respectively. Figure $3 \mathrm{c}$ represents the pristine $\mathrm{PC}$ sample at the same magnification with no micropatterning.

Detailed examination on SEM at higher resolution and magnification depict the presence of nanostructures on the PC surface modified at 600 EUV shots (Fig. 4). Surface morphology was also examined by the AFM. Figure 5a shows the AFM image of pristine PC sample while Figs. 5b,c and d shows PC sample irradiated with 100, 200, and 600 EUV shots, respectively. Results from both techniques demonstrate that the wall type nano and microstructures appeared on EUV treated surfaces which become more pronounced with an increase in the number of EUV shots. The micropattern introduced by EUV modification are similar to those which appeared after ultraviolet irradiation [15].

In order to characterize the roughness of the PC samples, 2D and 3D AFM measurements were obtained using semicontact mode. It can be observed from Fig. 6a and Fig. $7 \mathrm{~b}$ that wall type micropatterns up to several hun- 

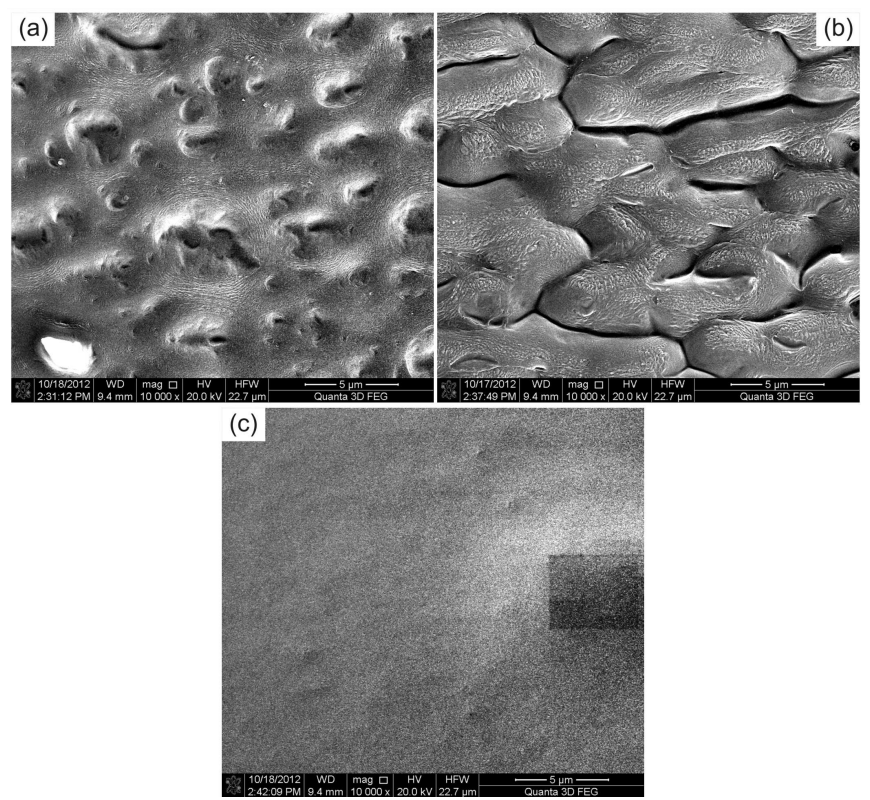

Fig. 3. SEM image of the PC sample irradiated with (a) 200 EUV shots, (b) 600 EUV shots, and (c) pristine $\mathrm{PC}$ at $10,000 \times$ magnification.

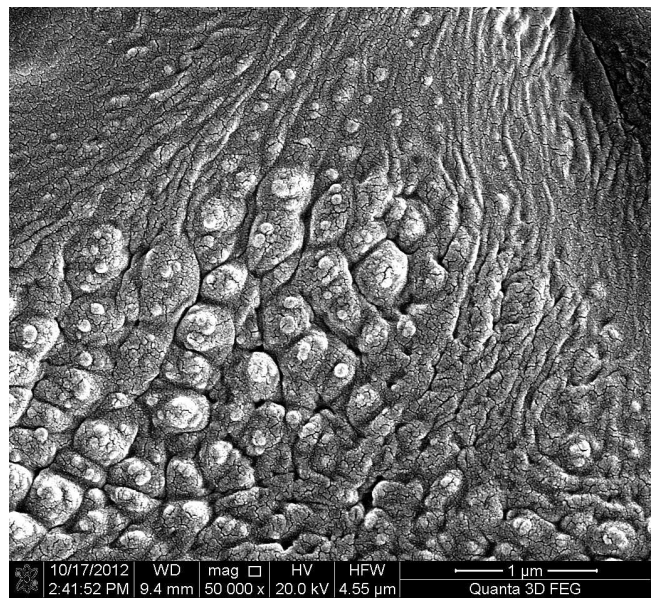

Fig. 4. SEM image of the PC sample irradiated with 600 EUV shots at $50 \mathrm{k} \times$ magnification.

dred nanometers in height appeared on the EUV treated PC sample surfaces. The reorganization of surface morphology and orientation of micropatterning is quite pronounced as shown in 3D images (see Figs. $6 \mathrm{~b}$ and $7 \mathrm{~b}$ ). As the sample holder was moving the patterns organized in quite well-ordered structural shapes which could help in adhesion and proliferation of different cell types under stochastic biological conditions. Roughness analysis of the AFM image of the EUV treated sample and the pristine sample was performed using the image analysis tool mentioned above.

Table I gives a summary of quantified results of roughness. Average surface roughness $\left(S_{\mathrm{a}}\right)$ and surface root mean square roughness $\left(S_{\mathrm{q}}\right)$ are the most interesting sta-
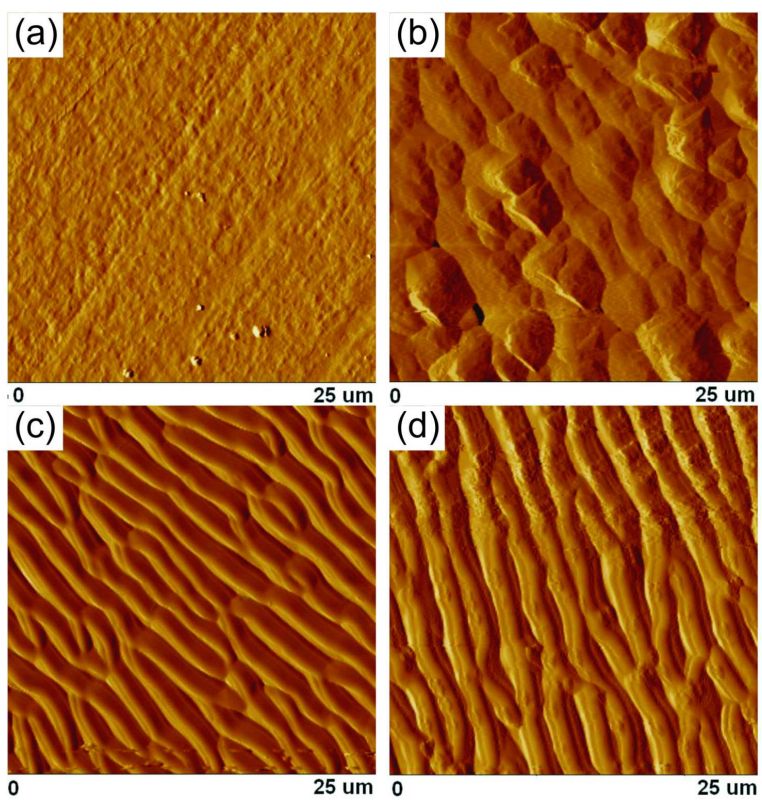

Fig. 5. AFM image of PC sample (a) pristine sample, (b) 100 EUV shots, (c) 200 EUV shots, and (d) 600 EUV shots.

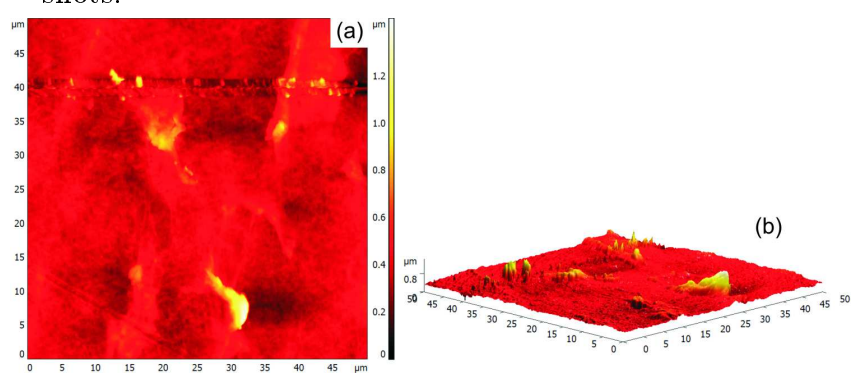

Fig. 6. AFM images of pristine PC sample at $50 \mu \mathrm{m}$ scale (a) 2D and (b) 3D.

tistical parameters to evaluate overall measure of the texture present on the surface (ISO norm 4287) [16, 17]. In order to characterize the uniformity of the texture over the surface area, the surface skewness $\left(S_{\mathrm{sk}}\right)$ can be calculated to evaluate the asymmetry of the amplitude distribution histogram. The $S_{\mathrm{a}}$ and $S_{\mathrm{q}}$ increased to more than 1.6 times in EUV treated sample as that of the pristine polymer sample. The surface skewness shifted from 0.63 (pristine sample) to 0.96 (EUV treated sample)

TABLE I

Roughness analysis of pristine and EUV modified PC samples.

\begin{tabular}{l|c|c}
\hline \hline \multicolumn{1}{c|}{ Parameters } & $\begin{array}{c}\text { pristine } \\
\text { PC } \\
\text { sample }\end{array}$ & $\begin{array}{c}\text { EUV } \\
\text { treated } \\
\text { sample }\end{array}$ \\
\hline maximum height recorded & $604 \mathrm{~nm}$ & $1430 \mathrm{~nm}$ \\
average surface roughness, $S_{\mathrm{a}}$ & $46 \mathrm{~nm}$ & $78 \mathrm{~nm}$ \\
root mean square roughness, $S_{q}$ & $66 \mathrm{~nm}$ & $106 \mathrm{~nm}$ \\
surface skewness, $S_{\mathrm{sk}}$ & 0.63 & 0.96
\end{tabular}




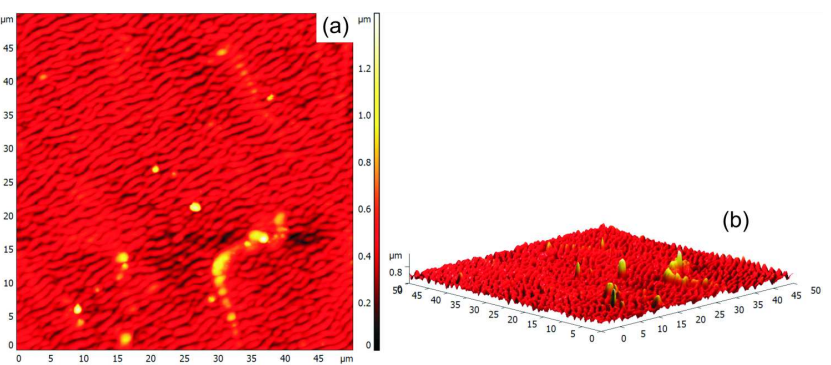

Fig. 7. AFM images of PC sample treated with 20 EUV shots (a) 2D and (b) 3D.

which indicates that extreme peaks are patterned on the surface after EUV surface modification. For such asymmetric distribution of roughness, leaning of mass distribution concentration towards the left represents quite a high quantitative difference between the two roughness measurements. The maximum peak height recorded in the pristine PC sample was about $604 \mathrm{~nm}$. The PC sample irradiated with 20 EUV shots contains structures up to $1430 \mathrm{~nm}$ high.
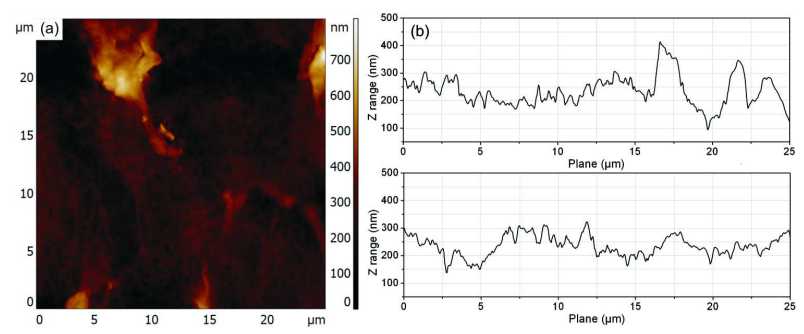

Fig. 8. AFM images of pristine PC samples at $25 \mu \mathrm{m}$ scale (a) 2D and (b) cross-sections.
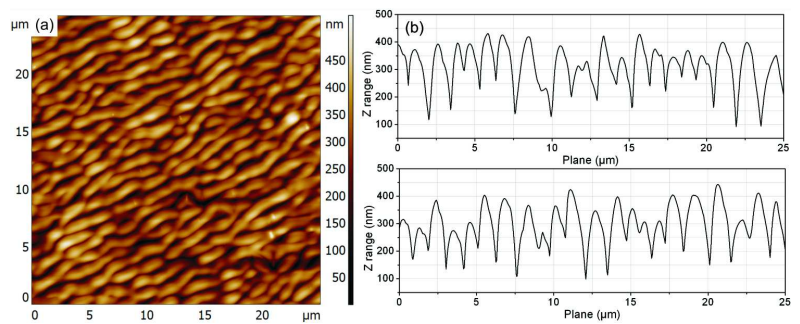

Fig. 9. AFM images of PC sample treated with 20 EUV shots $25 \mu \mathrm{m}$ scales (a) $2 \mathrm{D}$ and (b) cross-sections.

Cross-sectional views of pristine and EUV modified polymer surfaces demonstrate pronounced structured peaks with some degree of regularity (Fig. 8 and 9 ). The results of these statistical parameters demonstrate significant increment of surface roughness in EUV patterned surfaces as compared to that of the pure polymer sample.

Chemical modification in PC samples after EUV treatment was investigated by XPS. Low resolution XPS scans of pristine $\mathrm{PC}$ and EUV processed PC surfaces

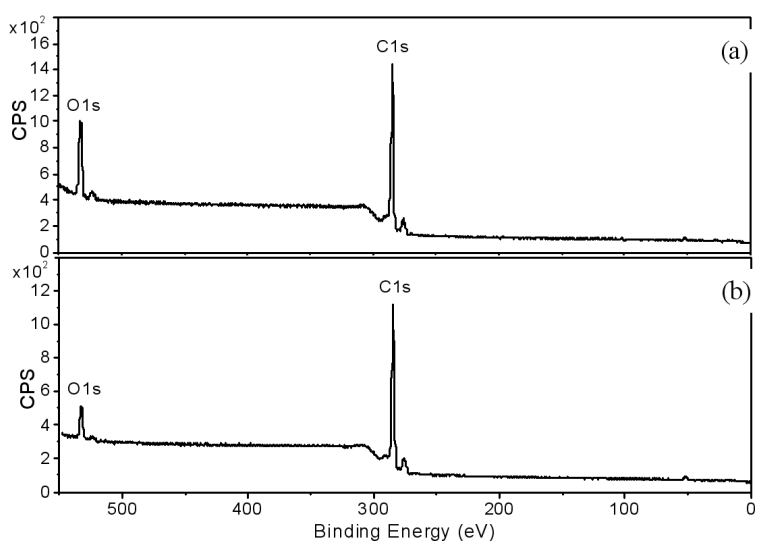

Fig. 10. XPS scans for polycarbonate (a) pristine sample, (b) modified sample irradiated with 20 EUV shots.

\section{TABLE II}

Summarized results from XPS spectra.

\begin{tabular}{c|c|c}
\hline \hline No. of EUV shots & Element & Atomic [\%] \\
\hline \multirow{2}{*}{ Pristine sample } & C $1 s$ & 83.47 \\
& O $1 s$ & 16.53 \\
\hline \multirow{2}{*}{20} & C $1 s$ & 91.97 \\
& O $1 s$ & 8.03
\end{tabular}

were acquired as shown in Fig. 10a and b, respectively. EUV treatment influences the $\mathrm{O} / \mathrm{C}$ ratio in $\mathrm{PC}$ sample. Table II summarizes the XPS results indicating a decrease in oxygen contents in the EUV modified surface. The decrease in oxygen contents consequently results in an increase of hydrophobicity which ultimately provide reduced wettability.

\section{Conclusion}

Upper layer surface physical and chemical properties of polycarbonate samples were successfully modified by EUV irradiation. Modified sample surfaces were characterized by SEM and AFM for surface morphology. Up to several hundred nanometers high wall-type micro- and nanostructures were formed on EUV modified PC samples similar to those introduced by ultraviolet radiation. Quantified roughness analysis showed that the surface roughness of EUV modified sample increase to double as that of pristine sample. Chemical analysis by X-ray photoemission spectra reveal a decreased oxygen contents in the EUV modified surfaces. Exclusion of oxygen from polar groups leads to a polymer with increased hydrophobicity. The wettability difference can be quantitatively measured by contact angle measurement in future studies. Due to the fundamental morphological and chemical changes to the EUV modified surfaces, this study has demonstrated the strong potential of EUV surface modification of polycarbonate to control the degree of the biocompatibility for certain biomedical engineering applications. 


\section{Acknowledgments}

Authors acknowledge financial support from the EU FP7 Erasmus Mundus Joint Doctorate Programme EXTATIC under framework partnership agreement FPA-2012-0033. With support from the 7th Framework Programme's Laserlab Europe project (No. 284464).

\section{References}

[1] J. Jagur-Grodzinski, React. Funct. Polym. 39, 99 (1999).

[2] L.G. Griffith, Acta Mater. 48, 263 (2000).

[3] B.L. Seal, T.C. Otero, A. Panitch, Mater. Sci. Eng. $R$, Reports 34, 147 (2001).

[4] A. Hadjizadeh, A. Ajji, M.N. Bureau, J. Mech. Behav. Biomed. Mater. 3, 574 (2010).

[5] T. Lippert, T. Nakamura, H. Niino, A. Yabe, Macromolecules 29, 6301 (1996).

[6] J. Heitz, J.D. Pedarnig, D. Bäuerle, G. Petzow, Appl. Phys. A 65, 259 (1997)

[7] Y. Liu, L. Liu, S. Fan, Appl. Phys. Lett. 86, 063105 (2005).

[8] P. Laurens, M. Ould Bouali, F. Meducin, B. Sadras, Appl. Surf. Sci. 154, 211 (2000).
[9] I.U. Ahad, A. Bartnik, H. Fiedorowicz, J. Kostecki, B. Korczyc, T. Ciach, D. Brabazon, J. Biomed Mater. Res. A (2013).

[10] B. Reisinger, M. Fahrner, I. Frischauf, S. Yakunin, V. Svorcik, H. Fiedorowicz, A. Bartnik, C. Romanin, J. Heitz, Appl. Phys. A 100, 511 (2010).

[11] I.U. Ahad, B. Budner, H. Fiedorowicz, A. Bartnik, D. Brabazon, Europ. Cells Mater. 26, 145 (2013).

[12] H. Fiedorowicz, A. Bartnik, Z. Patron, P. Parys, Appl. Phys. Lett. 62, 2778 (1993).

[13] A. Bartnik, H. Fiedorowicz, R. Jarocki, J. Kostecki, M. Szczurek, P. Wachulak, Nucl. Instrum. Methods phys. Res. A 647, 125 (2011).

[14] A. Bartnik, W. Lisowski, J. Sobczak, P. Wachulak, B. Budner, B. Korczyc, H. Fiedorowicz, Appl. Phys. A 109, 39 (2012).

[15] E. Arenholz, V. Svorcik, T. Kefer, J. Heitz, D. Bäuerle, Appl. Phys. A 53, 330 (1991).

[16] J. Jorgensen, K. Carneiro, L. Madsen, Nanotechnology 4, 152 (1993).

[17] P.D. Antonio, M. Lasalvia, G. Perna, V. Capozzi, Biochim. Biophys. Acta 1818, 3141 (2012). 